\title{
Inventory Control with Advance Demand Information When Demand is Intermittent
}

\author{
Borga Deniz \\ The Joseph W. Luter, III School of Business, Christopher Newport University \\ 1 Avenue of the Arts, Newport News, VA 23606, USA \\ Tel: 1-757-594-8915Ｅ-mail: borga.deniz@cnu.edu
}

Received: August 30, 2012

Accepted: September 21, 2012 Online Published: September 24, 2012

doi:10.5430/bmr.v1n4p35

URL: http://dx.doi.org/10.5430/bmr.v1n4p35

\begin{abstract}
In this paper we study a manufacturer-retailer relationship from the perspective of the manufacturer. The problem is managing the inventory of the manufacturer when Advance Demand Information (ADI) is available from the retailer. ADI allows the retailer to signal the manufacturer that there will be a demand, of the signaled size, in a future period. This is coupled with the situation where the manufacturer gets intermittent orders from the retailer. This intermittence may be because of the ordering policy of the retailer, or the nature of the product. Because of this demand structure the manufacturer's replenishment policy is assumed to be a "High-Low" base stock policy. A parsimonious Markov chain model of the intermittent demand with ADI is presented. We observe that a high-low policy is less costly than a one-parameter base-stock policy and that ADI increases the supply chain efficiency by reducing the cost of the manufacturer.
\end{abstract}

Keywords: Inventory management, Intermittent demand, Advance demand information

\section{Introduction}

As information technology develops, advance demand information (ADI) is becoming a major part of companies' demand structure. Because of ADI, customers can more easily place orders from the manufacturers, and this makes collaboration in the form of information sharing between manufacturer and customer more important in the competitive market. In addition, with the increase in internet usage, companies are also able to go directly to the consumer to get ADI. An important characteristic of many firms' demand structure is intermittence. Demand is intermittent if the orders from customers have a lumpy character, that is, after the manufacturer sees a demand, she is unlikely to see another demand soon. For instance, in the spare parts industry (e.g Xerox, GM) demand is intermittent. Spare parts are needed when machine breakdowns take place, and the component that needs to be replaced may be different at different incidents, leading to demand with high intermittence levels. Finally, might also become intermittent when customers order in batches, due to $(Q, R)$ or $(s, S)$ policies. Briefly, under these policies replenishment orders wait until the inventory level drops below a specific level ( $R$, in the first, $s$ in the latter) and then order is placed ( in the amount of Q in the first, $S$ - $s$ in the latter.) In this paper an inventory model with ADI, under intermittent demand is studied. In the model there is a manufacturer who satisfies or backorders the demand coming from the retailer. The manufacturer has a positive and constant manufacturing leadtime of $l$. When the customer demands an item she expects to have it immediately, so the manufacturer keeps an inventory of finished goods, in order not to suffer the backorder cost, which is linear. Inventory holding cost for the manufacturer is linear as well. Since the retailer also has her own customers, she might want to have the manufacturer start the production of items she needs in advance by giving the manufacturer ADI. This can help ensure that the manufacturer has them when she orders, and is particularly useful if $l$ is significant. Therefore the retailer is motivated to place advance orders if she is certain that she will need that amount in that particular future period. In this paper ADI is assumed to be firm, meaning, the retailer promises to buy the amounts she orders in advance. For orders that are placed further ahead than the manufacturer's leadtime, the order will be ready exactly when needed and no holding or penalty costs are charged to the manufacturer. For orders within the manufacturer's leadtime, any backorder will be satisfied sooner. If there was no advance ordering option, all these demands would be "normal" orders. (It is assumed that the retailer sees the exact same amount demand from her own customers, thus she will order this from manufacturer in both scenarios). This paper is on the positive effect of ADI on inventory holding and penalty costs via reduction of leadtime between parties. 
When the retailer is using an $(s, S)$ policy Gavirneni et al. (1999) showed that a state dependent base-stock policy is optimal. In order to make manufacturer's job simpler, instead of using a state dependent policy a high-low policy is used, which has two order-up-to levels, $S^{l}$ and $S^{\mathrm{h}}$. If the likelihood of getting a positive demand exceeds a threshold level, the manufacturer switches from the low order-up-to level $\left(S^{l}\right)$ to the higher one $\left(S^{h}\right)$. This switching decision is made based on the number of periods since the last demand $\left(\mathrm{L}_{\mathrm{n}}\right)$ (i.e. If $L_{n} \geq K$ then the order-up-to level is increased to $S^{h}$ ). This study explores appropriate levels of $S^{l}$ and $S^{h}$ and also the switching point $K$. Further, it explores how the optimal solution changes with problem parameter and compares the high-low policy with the 1-parameter base stock policy and the ADI model with non-ADI model.

In Section 2 there is a brief literature review of research on intermittent demand and ADI. In Section 3 modeling of the problem is presented. In Section 4 a numerical study and managerial insight is presented. Section 5 contains the conclusion and the future research directions.

\section{Brief Literature Review}

This paper is related to two streams of literature, one dealing with intermittent demand and the other with inventory management when ADI is available. Intermittent demand has been studied by many researchers; these studies are mainly on forecasting. The first important study on intermittent demand is by Croston (1972). This early paper highlights the inadequacies of available forecasting methods to estimate the demand pattern for items with intermittent orders. Croston suggests estimating the average interval between orders and the average size of an order when it occurs and to combine these statistics to give an unbiased estimate of the variability of the demand. Johnston and Boylan (1996) address the same problem. The order arrival process for the items with intermittent demand is modeled as Poisson stream and, therefore, an exponential distribution is used to model the inter-order interval. Order size is also another random variable. This paper aims to determine, under what conditions, intermittent demand requires its own model. Sani and Kingsman (1997) compare the forecasting methods for items with low or intermittent demand. Other studies on forecasting intermittent demand include papers by Syntetos and Boylan (2001, 2010), Ghobbar and Friend (2003), Willemain et al. (2004), Kalchschmidt et al. (2006), Altay et al. (2008), Syntetos et al. (2009), Teunter et al. (2010), Wallströma and Segerstedt (2010), and Rahman and Sarker (2012).

There are many studies dealing with inventory management with ADI, which is the other stream of literature. Heath and Jackson (1994) propose a general probabilistic model for modeling the evolution of demand forecasts, referred to as the Martingale Model of Forecast Evolution (MMFE). Gullu (1996), using MMFE, analyzes the effects of the forecasts on the production/ inventory system comparing the optimal ordering policy and the expected costs of the model that keeps forecasts with that of a comparable standard inventory model. Toktay and Wein (2001) use dynamically updated forecasts to determine production order releases for a capacitated system and propose an order release rule maintaining a "forecast-corrected" base-stock level. They obtain approximate closed-form expression for the optimal forecast-corrected base stock level. Ozer and Wei (2004) also use forecast updating models to obtain value of ADI and approximate the optimal base-stock levels.

Research which deals with state dependent policies where the demand is governed by either an external process or updated by advance demand information has been conducted by Song and Zipkin (1993), Sethi and Cheng (1997) and Gavirneni et al (1999). Hariharan and Zipkin (1995) were the first to study advance demand information in a multi-echelon setting. They study ADI in a continuous review series system and prove the optimality of base stock policies for a single stage system. Gallego and Ozer (2001) study multi-echelon inventory problems under advance order information and prove the optimality of state dependent echelon base stock policies for both finite and infinite horizon -discounted problems. They also show that the problem decomposes into single stage periodic review problems as in Clark and Scarf (1960). Wang and Toktay (2008) analyze inventory management with ADI and the possibility of flexible (early) delivery. For a centralized system, Ozer (2003) established inventory control policies for a supplier that replenishes the inventory of multiple retailers who obtain ADI. The author also provides closed-form solution to approximate the system wide inventory levels. Using such explicit solutions, and the replenishment policies, he quantities the joint role of risk pooling and ADI for a periodic review distribution system. The author also shows how ADI can be a substitute for replenishment leadtimes and inventory and how it enhances the outcome of delayed differentiation. Lu et al. (2003) analyze an assemble-to-order production system with advance demand information and random leadtime. Chen (2001) investigates market segmentation options in the presence of ADI. In his model a company offers different prices and delivery schedules; and segments the market based on different demand leadtimes. Thonemann (2002) and Zhu and Thonemann (2004) study the value of ADI which is received from a number of customers. Gayon et al. (2009) study imperfect ADI in systems with multiple demand classes and investigate how ADI can be utilized to improve production and inventory allocation decisions. 
The value of ADI has been investigated in capacitated production-inventory systems using queuing models, as well. Buzacott and Shanthikumar (1994), Karaesmen et al. (2002), and Karaesmen et al. (2003, 2004) study a single-stage make-to-stock queue with ADI, and analyze base-stock policies under various settings.

In this paper we study the relationship between a manufacturer and a retailer from the point of view of the manufacturer. We assume that in this relationship the strategic objectives of the manufacturer and the retailer are compatible. Furthermore, in a supply chain operational performance of companies can be affected by many uncertainties from the rest of the supply chain. Our model does not address those issues. Hendricks and Singhal (2005) study the relationship between supply chain glitches and operating performance of companies in the supply chain. In this empirical study they find that production and shipping delays in a supply chain decrease growth in sales and increase growth in cost and inventories.

We address a periodic-review, infinite horizon production system faced by a manufacturer who has the ability to get advance demand information while the demand is stochastic and intermittent. To our knowledge, this study is first on inventory management with ADI when demand is intermittent. In our model ADI allows the retailer to signal the manufacturer that there will be a demand, of the signaled size, in a future period. This is coupled with the situation where the manufacturer gets intermittent orders from the retailer. Due to the demand structure the manufacturer's replenishment policy is assumed to be a "High-Low" base-stock policy. With this policy, the manufacturer starts with a low order-up-to level, then switches to a higher order-up-to level if she does not see any demand for a certain number of consecutive periods and this number is called switching point. Therefore the parameters of the policy are: Low order-up-to level, high order-up-to level and switching point. We use a Markov chain model of the intermittent demand with ADI. We compare the high-low policy with a one-parameter base-stock policy in terms of cost and investigate the value of ADI. Therefore the specific research questions addressed in this paper are "What are the optimal parameters for the High-Low policy?", "How does this perform compared with a policy having a single base stock parameter?" and "What are the benefits of this information sharing for these two parties?".

\section{Modeling of the Problem}

In this paper a single item, single location, periodic review inventory model is considered. The manufacturer observes the following demand vector

$$
D_{n}=\left(d_{n, n}, d_{n, n+1}, \ldots, d_{n, n+H}\right)
$$

where $d_{n, n}$ is the order amount to be satisfied immediately, which will be called normal demand throughout this paper. The other components of the demand vector are considered as Advance Demand Information (ADI), which will be called advance demand throughout this paper. For example $d_{n, n+1}$ is the advance order amount revealed this period to be satisfied in the next period, and so forth. In period $n$, there is outstanding advance demand for the following $H$ periods (i.e. for $n+1, \ldots, n+H$ ) if

$$
\sum_{i=m-H}^{n} \mathrm{~d}_{\mathrm{i}, \mathrm{m}}>0
$$

for any

$$
m \in\{n+1, \ldots, n+H\}
$$

The demand vectors $D_{n}$ signal new demand registered in each period. For example if the demand information that the manufacturer gets from the retailer for a period (say period 5) is $D_{5}=(3,0,0,9)$ (i.e. demand to be satisfied this period is 3 units, and demand to be satisfied in $8^{\text {th }}$ period is 9 units); $D_{6}$ can be $(2,0,0,0)$, meaning the 9 units of order for $8^{\text {th }}$ period does not appear in this demand vector. Therefore, in order to find the demand for a particular period the components of the vectors which have that particular period in their range have to be added. Thus the demand that has to be fulfilled in period $n$ is as follows:

$$
\sum_{i=n-H}^{n} \mathrm{~d}_{\mathrm{i}, \mathrm{n}} .
$$

The model assumes that an advance demand is firm, that is, the retailer will not renege on this order. $H$ is the $A D I$ horizon for the problem, meaning the retailer can order at most $H$ period ahead. The manufacturer has a deterministic leadtime, $l$. That is, when the retailer places orders in period $n$, her order is going to be satisfied no later than period $n$ $+l+1$. For example, if there is no leadtime (i.e. $l=0$ ), and the retailer places an order now, the order arrives in the next period. In this paper the upper stage of the supply chain is called as the manufacturer, and it is assumed that she 
has no capacity constraint. Leadtime is a manufacturing leadtime. If the upper stage does not do manufacturing and just ordering from an upper supplier with ample stock then the leadtime would be ordering leadtime.

The sequence of events in a period is as follows: First, replenishment arrives at the manufacturer. Second, demand occurs. Demand is immediately satisfied if possible, otherwise it is fully backlogged. Demand is satisfied from on hand inventory with priority of existing backorders.

A penalty cost of $b$ for backorders and holding cost of, $h$, for any inventory left on hand are incurred. Both costs are per unit, per period. Finally, a new order is placed, which may include any advance demand. In the model, the manufacturer keeps a certain amount of inventory ( i.e. base stock) and orders the exact amount of the total demands (normal and advance), that she sees in that period. This kind of ordering policy can be called a state dependent base stock policy where the state is the amount of the outstanding advance orders from the retailer.

An important point is the following: For an advance demand $d_{n, n+y}$, if $y>l$, no cost is incurred. In such a case, demand can be satisfied just-in-time without incurring any penalty or holding costs; therefore this case is not considered here.

The notation in this paper is as follows:

$I_{n}=$ On-hand inventory at the beginning of the period $n$. This is the inventory that is physically on the shelf, which cannot be negative.

$L_{n}=$ Number of periods since last order in period $n . L_{n}$ is 1 if a demand occurred in period $n-1$.

$Q_{n}=$ Order amount which will arrive by the beginning of period $n+l+1$.

$B_{n}=$ Backorder in period $n$.

$N I_{n}=$ Net inventory in period $n$, which is inventory on hand minus backorder,

$$
N I_{n}=I_{n}-B_{n} .
$$

$I P_{n}=$ Inventory position, which is net inventory plus amount of the order in period $n$,

$$
I P_{n}=I_{n}-B_{n}+Q_{n} .
$$

$I I P_{n}=$ Informed inventory position, which is inventory position minus the advance demands which have been signaled but have not been materialized,

\subsection{Stationary Demand Case}

$$
I I P_{n}=I P_{n}-\sum_{j=n+1}^{n+H} \sum_{i=j-H}^{n} d_{i, j} .
$$

In a stationary demand process, the probability of having a positive demand and the distribution of demand do not change over time; they are the same in every period.

Let $p_{i}$ be the probability of being informed of a positive demand $i-1$ periods ahead. That is,

$$
p_{i}=\operatorname{Pr}\left(d_{n, n+i-1}>0\right)
$$

for any period $\mathrm{n}$. For example, $p_{1}$ is the probability of having normal positive demand this period. The demand process is stationary as this probability is not dependent on $n$ or $L_{n}$. In this case the probability of having no advance demand information can be written as follows:

$$
1-\sum_{i=1}^{H+1} p_{i}
$$

The demand itself can be distributed with any distribution. Gallego and Ozer (2001) show that for this case optimal policy is state dependent base stock. This policy can be called a base stock policy based on IIP. Gallego and Ozer (2001) do not show how to get the optimal base stock parameter.

The expected cost per period under this model is as follows:

where,

$$
G(I I P)=h E[I I P-D]^{+}+b E[I I P-D]^{-}
$$

$D=$ Demand over the leadtime, 


$$
D=\sum_{i=1}^{l+1} \sum_{j=i}^{l+1} d_{i, j}
$$

\subsection{Intermittent Demand Case}

In the model the demand is not stationary; it is dependent on the number of periods since the last demand. Specifically, the probability of having nonzero demand of any type in a period increases as the number of periods since the last positive demand increases. To model this let the probability of having information of a positive demand $\mathrm{i}-1$ periods ahead be as follows:

$$
p_{i}^{(k)}=1-\left[\left(1-p_{i}\right) \theta^{k-1}\right]
$$

for all $i \in[1, H+1]$, where $\theta$ is a positive real number less than 1 and $k$ is the number of periods since last demand (the same as $L_{n}$ ). It can be observed that if $\theta=1$ then the demand process is stationary; and when $\theta=0$ there is positive demand for every period.

Gavirneni et al (1999) found that, under intermittent demand, the optimal policy is state dependent base stock policy. In their paper intermittence is due to $(S, S)$ policy of the retailer, and there is no advance demand information available. There the manufacturer's optimal policy is a state dependent base stock policy with state $L_{n}$. That is, for every different value of $L_{n}$ (number of periods since the last demand), the order-up-to levels change; as $L_{n}$ increases, the order-up-to level also increases. However, such a policy is very hard to apply to practice, owing to the large amount of inventory parameters. Therefore a policy which is more applicable is studied: a "High-Low Policy" that has only two order-up-to levels. The low level is $S^{l}$, and if there is no order from the retailer for $K$ periods in a row, the manufacturer switches to the high order-up-to level, $S^{h}$.

A Markov chain can represent this situation. To ensure a parsimonious exposition to illustrate the model, the pairs of $S^{l}$ and $S^{h}$ are limited so that,

$$
S^{h}-S^{l} \leq \min d_{i, j},
$$

for any $i$ and $j$. This guarantees that, if a demand is observed when IIP $=S^{h}$, the next IIP level is going to be $S^{l}$. Therefore the IIP levels which are between $S^{h}$ and $S^{l}$ may be eliminated, saving a dimension in the state space of the Markov chain. This assumption reduces the numerical work that is required to find the stationary probabilities of the states. If this assumption is not made the methods in this paper are still applicable, but computations are more intensive. Rather than solving a simple set of balance equations as below matrix geometric methods may need to be applied. The state of this Markov chain is the number of periods since last demand. : (1), (2), .., , $K),(K+1),(K+$ $2), \ldots$ In the next period the state number is going to increase by one if there is no demand in the period, and is going to be 1 otherwise.

Let $R^{(k)}$ be the probability of not having any positive demand (advance or normal) given that number of periods since last positive demand is $k$. Therefore,

$$
\begin{gathered}
R^{(k)}=\prod_{i=1}^{H+1}\left(1-p_{i}^{(k)}\right), \\
=\prod_{i=1}^{H+1}\left(1-1+\left[\left(1-p_{i}\right) \theta^{k-1}\right]\right), \\
=\prod_{i=1}^{H+1}\left(1-p_{i}\right) \theta^{k-1}, \\
=R^{(1)} \theta^{(H+1)(k-1)} .
\end{gathered}
$$

The transition probabilities are as follows: Let $P_{i, j}$ be the transition probability from state $i$ to state $j$. Then,

for all $i \in\{1,2, \ldots\}$.

$$
\begin{gathered}
P_{i, i+1}=R^{(i)}, \\
P_{i, 1}=1-R^{(i)},
\end{gathered}
$$

This Markov chain is positive recurrent ergodic as long as some $p_{i}>0$ and $\theta \leq 1$. Therefore the stationary probabilities of the Markov chain, which are known to exist can be found by solving the balance equations:

$$
\begin{gathered}
\pi_{2}=\pi_{1} R^{(1)}, \\
\pi_{3}=\pi_{2} R^{(2)}, \\
\\
\cdots
\end{gathered}
$$




$$
\pi_{i}=\pi_{i-1} R^{(i-1)}
$$

and,

These yield:

$$
\pi_{1}+\pi_{2}+\pi_{3}+\cdots=1 .
$$

for all $\mathrm{j} \in\{2,3, \ldots\}$.

$$
\begin{gathered}
\pi_{1}=\frac{1}{\sum_{i=0}^{\infty}\left(R^{(1)}\right)^{i} \theta^{\frac{(H+1)(i-1) i}{2}}}, \\
\pi_{j}=\pi_{1} \prod_{i=1}^{j-1} R^{(i)},
\end{gathered}
$$

The cost of a state consists of linear penalty and holding cost over the leadtime period. At the beginning of the leadtime period, the IIP level is either $S^{h}$ or $S^{l}$, and at the end of this period, the on hand inventory would be the IIP level minus the leadtime demand. Hence the cost of a state can be written as follows:

$$
\begin{aligned}
G[k] & =h E[I I P(k)-D(k)]^{+}+b E[I I P(k)-D(k)]^{-}, \\
& =h I I P(k)-h E[D(k)]+(h+b) E[I I P(k)-D(k)]^{-}, \\
& =h I I P(k)-h E[D(k)]+(h+b) \sum_{i=I I P(k)}^{D_{\max }}(i-I I P(k)) \operatorname{Pr}(D=i),
\end{aligned}
$$

where,

$$
I I P(k)= \begin{cases}S^{l} \text { If } k \leq K, \\ S^{h} \text { If } k>K,\end{cases}
$$

and $D(k)$ is demand over leadtime,

$$
D(k)=\sum_{i=1}^{l+1} \sum_{j=1}^{l+1} d(k)_{i, j}
$$

where,

$$
d(k)_{i, j} \sim \begin{cases}0 & \text { with probability } 1-p_{j-i+1}^{(k)} \\ d & \text { with probability } p_{j-i+1}^{(k)} .\end{cases}
$$

And $d$ is the distribution of demand regardless of the number of periods since last positive demand. For simplicity, it is taken identical for all the normal and advance parts of demand vector.

Given that the stationary probability and expected cost of each state is available, then total expected cost is as follows:

$$
\sum_{i=1}^{\infty} \pi_{i} G[i]
$$

This cost can be found numerically, and eventually optimal parameters $\left(K ; S^{l} ; S^{h}\right)$ can be found.

Proposition 3.1 Cost function is non-decreasing in number of indices for the state dependent base-stock policies.

Proof : Let policy $N$ have $n$ order-up-to levels (like high-low policy has two order-up-to levels). Let the optimal values for these be $S^{1}, S^{2}, \ldots, S^{n}$ with the relation

$$
S^{1} \leq S^{2} \leq \cdots \leq S^{n}
$$

and the corresponding minimum cost of $C_{N}$. Let policy $N+1$ be the one with $n+1$ indices for the order up to levels. Policy $N+1$ has a cost of $C_{N}$ with order up to levels $S^{1}, S^{2}, \ldots, S^{n}, S^{n+1}$ where $S^{n}=S^{n+1}$. Therefore policy $N+1$ cannot do worse than policy $N$ (i.e. $C_{N+1} \leq C_{N}$ ).

\section{Numerical Study}

For the numerical study the same discrete uniform distribution is used for all the components of the demand vector for simplicity. The numerical study is done to see results for a sample problem. The values in Table 1 are used for the numerical example. Any realized demand is distributed by discrete uniform distribution between 4 and 7 . To see the effect of intermittence the following values for $\theta$ are used: $0.99,0.975,0.95,0.9,0.75,0.5$ and 0.1 . 
The average number of consecutive demandless periods increases as the intermittence increases (i.e. $\theta$ increases). When the demand is highly intermittent $(\theta=0.99)$, the demand occurs every 4.44 periods, on the average. Demand occurrence becomes more frequent with smaller $\theta$ as it is shown in Table 2 .

The stationary distribution of the states become negligible after only a few states. (The stationary distributions for $\theta=$ 0.99 and 0.1 are tabulated in Table 3.) Therefore, even if there are infinite number of states in the model's Markov chain, only a small number of them are used in the numerical experiments.

The search is done over $K=1$ to $7, S^{l}=1$ to 20 and $S^{h}=S^{l}$ to $S^{l}+4$. (The search is for $S^{h}=S^{l}$ to $S^{l}+4$ for every $S^{l}$ because of the assumption that $S^{h}-S^{l} \leq \min d=4$ )

For example for $\theta=0.99$, the optimal high-low policy parameters are: $S^{l}=1 ; S^{h}=5$ and $K=4$, (i.e. the manufacturer should keep inventory of 1 unit when she sees a demand, and if she does not see any demand for 4 periods in a row, she should order 4 more units.)

A summary of optimal high-low parameters for various $\theta$ values is in Table 4. It seems that the

$$
\min d \geq S^{h}-S^{l}
$$

constraint is binding, and neither cost nor service level is monotone in intermittence.

\subsection{High-Low Policy vs. One-Parameter Policy}

In order to see the benefit of the high-low policy over the one-parameter policy, a comparison is carried out in terms of the optimal costs and corresponding fill rates. As it is seen in Table 5, the high-low policy is less costly than the single parameter base stock policy for all intermittence levels. It appears that high-low policy is most valuable when $\theta$ is very high or very low, besides it also can decrease cost and increase fill rate.

\subsection{Cost Reduction of Manufacturer due to ADI}

Retailer shares her information with the manufacturer, as a result, manufacturer reduces her costs due to the leadtime decrease by the advance demand information. Table 6 shows the cost savings of the manufacturer for different $\theta$ values; the table demonstrates that cost savings due to ADI are more significant for less intermittent cases.

\subsection{Is the Retailer Happy about the ADI?}

Table 7 shows the fill rates of the manufacturer for the optimal high-low policies with and without ADI. As demand gets more intermittent (i.e. higher $\theta$ ), fill rate decreases under ADI; however when ADI is not available we do not observe such a relationship. Strictly speaking we cannot conclude that sharing (or not sharing) information gets the retailer a better service level from the manufacturer, it changes under different intermittence levels. But for higher intermittence levels it looks like the retailer receives a better service level when ADI is not shared. Table 8 provides a cost comparison between ADI and non-ADI cases when there is a contract between retailer and manufacturer specifying a 95\% service level (fill rate).

\subsection{Value of ADI for Various Cost Parameters}

Penalty cost for backlogging is $b$ and cost of holding inventory is $h$; and in most cases backlogging is more costly than holding. These costs may differ for different products and customers who are buying the product. In order to see the effect of $b / h$ ratio on the cost and fill rate change due to ADI $h$ is fixed at 1 and $b$ is given several values. Table 9 shows the minimum costs and corresponding fill rates for various $b$ values and the effect of ADI ( $\theta$ is taken as 0.90.) There is a dramatic increase in cost savings when the ratio increases from 0.1 to 0.5 but for larger values of the ratio the savings show moderate fluctuation.

\section{Conclusion and Future Research Directions}

In this study a Markov chain model is developed to find the optimal parameters of the high-low policy for the manufacturer. We observed that a high-low policy is less costly than a one-parameter base stock policy. Another result of this study is that ADI increases the supply chain efficiency by reducing the cost of the manufacturer. Information that is shared by the retailer helps the manufacturer provide higher service level to the retailer with lesser operating costs. The Markov chain model in this paper assumes that, if a demand occurs when the manufacturer is in a high state, it will be large enough (namely, at least in the amount of $S^{h}-S^{l}$ ) to make the next state a low state eliminating intermediate states. As a future work, the assumption can be relaxed. The $\theta$ used in the model provides the intermittent nature of the demand and it is taken as a constant as the number of periods since the last demand $\left(L_{n}\right)$ increases. $\theta$ can be a function of $L_{n}$ and its effects can be studied. Also, the mathematical formulation presented in this study can be extended by adding supply chain glitches into the model. 


\section{References}

Altay, N., Rudisill, F., \& Litteral, L. A. (2008). Adapting Wright's modification of Holt's method to forecasting intermittent demand. International Journal of Production Economics, 111(2), 389 - 408. http://dx.doi.org/10.1016/j.ijpe.2011.08.002

Buzacott, J. A. \&, Shanthikumar, J. G. (1994). Safety stock versus safety time in MRP controlled production systems. Management Science, 40, 1678-1689. http://dx.doi.org/10.1287/mnsc.40.12.1678

Chen, F. (2001). Market segmentation, advanced demand information, and supply chain performance. Manufacturing Service Operations Management, 3(1), 53-67. http://dx.doi.org/10.1287/msom.3.1.53.9993

Clark, A.J., \& Scarf, H. (1960). Optimal policies for a multi-echelon inventory problem. Management Science 6, 475-490. http://dx.doi.org/10.1287/mnsc.1040.0265

Croston , J.D. (1972). Forecasting and stock control for intermittent demands. Operations Research Quarterly, 23(3), 289-303. http://dx.doi.org/10.1057/jors.1972.50

Gallego, G. \& Ozer, O. (2001). Integrating replenishment decisions with advance demand information. Management Science, 47, 1344-1360. http://dx.doi.org/10.1287/mnsc.47.10.1344.10261

Gavirneni, S., Kapuscinski, R. \& Tayur S. (1999). Value of information in capacitated supply chains. Management Science 45(1), 16-24. http://dx.doi.org/10.1287/mnsc.45.1.16

Gayon, J-P., Benjaafar, S. \& de Vericourt, F. (2009). Using imperfect advance demand information in production-inventory systems with multiple customer classes. Manufacturing \& Service Operations Management, 11(1), 128-143. http://dx.doi.org/10.1287/msom.1070.0201

Ghobbar, A.A., \& Friend, C.H. (2003). Evaluation of forecasting methods for intermittent parts demand in the field of aviation: a predictive model. Computers \& Operations Research, 30, 2097-2114. http://dx.doi.org/10.1016/S0305-0548(02)00125-9

Gullu, R. (1996). On the value of information in dynamic production/inventory problems under forecast evolution. Naval Research Logistics, 289-303. http://dx.doi.org/10.1002/(SICI)1520-6750(199603)43:2<289::AID-NAV8>3.0.CO;2-6

Hariharan, R. \& Zipkin, P. (1995). Customer order information, leadtimes, and inventories. Management Science, 41, 1599-1607. http://dx.doi.org/10.1287/mnsc.41.10.1599

Heath, D. \& Jackson, P. (1994). Modeling the evolution of demand forecasts with application to safety stock analysis in production/distribution systems . IIE Transactions, 26, 17-30. http://dx.doi.org/10.1080/07408179408966604

Hendricks K. B. \& Singhal V. R. (2005). Association between supply chain glitches and operating performance. Management Science, 51(5), 695-711. http://dx.doi.org/10.1287/mnsc.1040.0353

Johnston, F.R, \& Boylan, J.E. (1996). Forecasting for items with intermittent demand. Journal of Operational Research Society, 47(1), 113-121. http://dx.doi.org/10.1057/jors.1996.10

Kalchschmidt, M., Verganti, R. \& Zotteri, G. (2006). Forecasting demand from heterogeneous customers. International Journal of Operations \& Production Management, 26(6), 619-638. http://dx.doi.org/10.1108/01443570610666975

Karaesmen, F., Buzacott, J. A. , \& Dallery, Y. (2002). Integrating advance order information in make-to-stock production systems. IIE Transactions, 34(8), 649-662. http://dx.doi.org/10.1023/A:1014925525185

Karaesmen, F., Liberopoulos G., \& Dallery Y. (2003). Production/ inventory control with advance demand information. In J. G. Shanthikumar, D. D. Yao, W. H. M. Zijm, (Eds.) Stochastic Modeling and Optimization of Manufacturing Systems and Supply Chains, International Series in Operations Research \& Management Science, Vol. 63. (pp. 243-270). Boston: Kluwer Academic Publishers. http://dx.doi.org/10.1007/978-1-4615-0373-6_10

Karaesmen, F., Liberopoulos G., \& Dallery Y. (2004). The value of advance demand information in production / inventory systems. Annals of Operations Research, 126(1-4), 135-157. http://dx.doi.org/10.1023/B:ANOR.0000012278.41301.24

Lu, Y., Song, J. S. \& Yao D. D.. (2003). Order fill rate, leadtime variability, and advance demand information in an assemble-to-order system. Operations Research, 51(2), 292-308. http://dx.doi.org/10.1287/opre.51.2.292.12781

Ozer, O. (2003). Replenishment strategies for distribution systems under advance demand information. Management Science, 49(3), 255-272. http://dx.doi.org/10.1287/mnsc.49.3.255.12738

Ozer, O., \& Wei W. (2004). Inventory control with limited capacity and advance demand information . Operations Research, 52(6), 988-1000. http://dx.doi.org/10.1287/opre.1040.0126 
Rahman, M. A., \& Sarker, B. R. (2012). A Bayesian approach to forecast intermittent demand for seasonal products. International Journal of Industrial and Systems Engineering, 11(1), 137-153. http://dx.doi.org/10.1504/IJISE.2012.046660

Sani, B., \& Kingsman, B.G. (1997). Selecting the best periodic inventory control and demand forecasting methods for low demand items. Journal of Operational Research Society, 48(7), 700-713. http://dx.doi.org/10.1057/palgrave.jors.2600418

Sethi, P. S. \& Cheng F. (1997). Optimality of (s, S) policies in inventory models with Markovian demand. Operations Research. 931-939. http://dx.doi.org/10.1287/opre.45.6.931

Song, J. S., \& Zipkin P. (1993). Inventory control in fluctuating demand environment. Operations Research, 41, 351-370. http://dx.doi.org/10.1287/opre.41.2.351

Syntetos, A. A., \& Boylan, J. E. (2001). On the bias of intermittent demand estimates. International Journal of Production Economics, 71(1-3), 457 - 466. http://dx.doi.org/10.1016/S0925-5273(00)00143-2

Syntetos, A. A., \& Boylan, J. E. (2010). On the variance of intermittent demand estimates. International Journal of Production Economics, 128(2), 546 - 555. http://dx.doi.org/10.1016/j.ijpe.2010.07.005

Syntetos, A. A., Nikolopoulos, K., Boylan, J. E., Fildes, R., \& Goodwin P. (2009). The effects of integrating management judgement into intermittent demand forecasts. International Journal of Production Economics, 118(1), 72 - 81. http://dx.doi.org/10.1016/j.ijpe.2008.08.011

Teunter, R. H., Syntetos, A. A., \& Babai, M. Z. (2010). Determining order-up-to levels under periodic review for compound binomial (intermittent) demand. European Journal of Operational Research 203(3), 619-624. http://dx.doi.org/10.1016/j.ejor.2009.09.013

Thonemann, U. W. (2002). Improving supply-chain performance by sharing advance demand information. European Journal of Operational Research 142(1), 81-107. http://dx.doi.org/10.1016/S0377-2217(01)00281-8

Toktay, L. B., \& Wein L. M. (2001). Analysis of a forecasting-production-inventory system with stationary demand. Management Science, 47(9), 1268-1281. http://dx.doi.org/10.1287/mnsc.47.9.1268.9787

Wallström, P., \& Segerstedt A. (2010). Evaluation of forecasting error measurements and techniques for intermittent demand. International Journal of Production Economics, 128(2), 625-636. http://dx.doi.org/10.1016/j.ijpe.2010.07.013

Wang, T., \& Toktay, B. L. (2008). Inventory management with advance demand information and flexible delivery. Management Science, 54, 716-732. http://dx.doi.org/10.1287/mnsc.1070.0831

Willemain, T.R., Smart, C.N., \& Schwarz, H. F. (2004). A new approach to forecasting intermittent demand for service parts inventories. International Journal of Forecasting, 20(3), 375-387. http://dx.doi.org/10.1016/S0169-2070(03)00013-X

Zhu, K., \& Thonemann, U. W. (2004). Modeling the benefits of sharing future demand information. Operations. Research, 52(1), 136-147. http://dx.doi.org/10.1287/opre.1030.0061

Table 1. Parameter values for the numerical study

\begin{tabular}{|c|c|}
\hline$l$ & 2 \\
\hline$H$ & 2 \\
\hline$p_{1}$ & 0.05 \\
\hline$p_{2}$ & 0.03 \\
\hline$p_{3}$ & 0.01 \\
\hline$h$ & 1 \\
\hline$b$ & 2 \\
\hline
\end{tabular}

Table 2. Average number of consecutive periods without any demand

\begin{tabular}{|c|c|}
\hline$\theta$ & Avg. number of periods without any demand \\
\hline 0.99 & 4.44 \\
\hline 0.975 & 3.33 \\
\hline 0.95 & 2.70 \\
\hline 0.9 & 2.22 \\
\hline 0.75 & 1.78 \\
\hline 0.5 & 1.56 \\
\hline 0.1 & 1.48 \\
\hline
\end{tabular}


Table 3. Stationary distribution of the Markov chain

\begin{tabular}{|c|c|c|}
\hline & \multicolumn{2}{|c|}{$\theta$} \\
\cline { 2 - 3 } & 0.99 & 0.1 \\
\hline$\pi_{1}$ & 0.173991 & 0.522707 \\
\hline$\pi_{2}$ & 0.158729 & 0.476858 \\
\hline$\pi_{3}$ & 0.140506 & 0.00044 \\
\hline$\pi_{4}$ & 0.12068 & $3.97 \mathrm{E}-10$ \\
\hline$\pi_{5}$ & 0.100573 & $3.62 \mathrm{E}-19$ \\
\hline$\pi_{6}$ & 0.081327 & $3.30 \mathrm{E}-31$ \\
\hline$\pi_{7}$ & 0.063811 & 0 \\
\hline$\pi_{8}$ & 0.04858 & 0 \\
\hline$\pi_{9}$ & 0.035886 & 0 \\
\hline$\pi_{10}$ & 0.025722 & 0 \\
\hline$\pi_{11}$ & 0.017889 & 0 \\
\hline$\pi_{12}$ & 0.012072 & 0 \\
\hline$\pi_{13}$ & 0.007904 & 0 \\
\hline$\pi_{14}$ & 0.005022 & 0 \\
\hline$\pi_{15}$ & 0.003096 & 0 \\
\hline$\pi_{16}$ & 0.001852 & 0 \\
\hline$\pi_{17}$ & 0.001075 & 0 \\
\hline$\pi_{18}$ & 0.000605 & 0 \\
\hline$\pi_{19}$ & 0.000331 & 0 \\
\hline$\pi_{20}$ & 0.000175 & 0 \\
\hline$\pi_{21}$ & $9.02 \mathrm{E}-05$ & 0 \\
\hline$\pi_{22}$ & $4.50 \mathrm{E}-05$ & 0 \\
\hline$\pi_{23}$ & $2.18 \mathrm{E}-05$ & 0 \\
\hline$\pi_{24}$ & $1.02 \mathrm{E}-05$ & 0 \\
\hline$\pi_{25}$ & $4.67 \mathrm{E}-06$ & 0 \\
\hline & & \\
\hline
\end{tabular}

Table 4. Optimal values for various intermittence levels

\begin{tabular}{|c|c|c|c|c|c|c|c|}
\hline & \multicolumn{7}{|c|}{$\theta$} \\
\cline { 2 - 8 } & 0.99 & 0.975 & 0.95 & 0.90 & 0.75 & 0.50 & 0.10 \\
\hline $\mathrm{S}^{1}$ & 1 & 1 & 2 & 5 & 7 & 10 & 15 \\
\hline $\mathrm{S}^{\mathrm{h}}$ & 5 & 5 & 6 & 7 & 11 & 14 & 19 \\
\hline $\mathrm{K}$ & 4 & 1 & 1 & 1 & 1 & 1 & 1 \\
\hline Cost & 4.12 & 4.64 & 5.09 & 5.59 & 6.15 & 6.45 & 5.73 \\
\hline Fill rate & $82.50 \%$ & $86.70 \%$ & $86.50 \%$ & $89.20 \%$ & $62.00 \%$ & $92.70 \%$ & $94.70 \%$ \\
\hline
\end{tabular}

Table 5. High-low policy vs. 1-parameter policy

\begin{tabular}{|c|c|c|c|c|}
\hline \multirow{2}{*}{$\theta$} & \multicolumn{2}{|c|}{ Cost } & \multicolumn{2}{c|}{ Fill rate } \\
\cline { 2 - 5 } & High-Low & 1-Parameter & High-Low & 1-Parameter \\
\hline 0.99 & 4.12 & 4.43 & $82.5 \%$ & $89.2 \%$ \\
\hline 0.975 & 4.64 & 4.86 & $86.7 \%$ & $90.6 \%$ \\
\hline 0.95 & 5.09 & 5.26 & $86.5 \%$ & $87.5 \%$ \\
\hline 0.9 & 5.59 & 5.79 & $89.2 \%$ & $87.8 \%$ \\
\hline 0.75 & 6.15 & 6.82 & $92.0 \%$ & $90.0 \%$ \\
\hline 0.5 & 6.45 & 7.59 & $92.7 \%$ & $86.3 \%$ \\
\hline 0.1 & 5.73 & 7.40 & $94.7 \%$ & $94.0 \%$ \\
\hline
\end{tabular}


Table 6. Cost comparison between ADI and non-ADI cases for the manufacturer

\begin{tabular}{|c|c|c|c|}
\hline \multirow{2}{*}{$\theta$} & \multicolumn{2}{|c|}{ Optimal high-low policy cost } & $\begin{array}{c}\text { Cost savings } \\
\text { due to ADI }\end{array}$ \\
\cline { 2 - 3 } & ADI & Non-ADI & $15.4 \%$ \\
\hline 0.99 & 4.12 & 4.87 & $16.1 \%$ \\
\hline 0.975 & 4.64 & 5.53 & $18.7 \%$ \\
\hline 0.95 & 5.09 & 6.26 & $22.1 \%$ \\
\hline 0.9 & 5.59 & 7.18 & $29.6 \%$ \\
\hline 0.75 & 6.15 & 8.74 & $31.7 \%$ \\
\hline 0.5 & 6.45 & 9.45 & $43.9 \%$ \\
\hline 0.1 & 5.73 & 10.21 & \\
\hline
\end{tabular}

Table 7. Fill rate of manufacturer

\begin{tabular}{|c|c|c|}
\hline \multirow{2}{*}{$\theta$} & \multicolumn{2}{|c|}{ Fill rate } \\
\cline { 2 - 3 } & ADI & Non-ADI \\
\hline 0.99 & $82.50 \%$ & $83.00 \%$ \\
\hline 0.975 & $86.70 \%$ & $89.00 \%$ \\
\hline 0.95 & $86.50 \%$ & $88.80 \%$ \\
\hline 0.9 & $89.20 \%$ & $90.80 \%$ \\
\hline 0.75 & $92.00 \%$ & $90.90 \%$ \\
\hline 0.5 & $92.70 \%$ & $92.80 \%$ \\
\hline 0.1 & $94.70 \%$ & $89.30 \%$ \\
\hline
\end{tabular}

Table 8. Costs for ADI and non-ADI models under the 95\% service level

\begin{tabular}{|c|c|c|c|}
\hline \multirow{2}{*}{$\theta$} & \multicolumn{2}{|c|}{ High-low policy cost } & \multirow{2}{*}{$\begin{array}{c}\text { Cost savings } \\
\text { due to ADI }\end{array}$} \\
\cline { 2 - 3 } & ADI & Non-ADI & $11.5 \%$ \\
\hline 0.99 & 4.69 & 5.3 & $16.9 \%$ \\
\hline 0.975 & 5.07 & 6.1 & $19.0 \%$ \\
\hline 0.95 & 5.57 & 6.88 & $24.4 \%$ \\
\hline 0.9 & 5.89 & 7.79 & $28.9 \%$ \\
\hline 0.75 & 6.58 & 9.26 & $33.2 \%$ \\
\hline 0.5 & 6.52 & 9.76 & $37.6 \%$ \\
\hline 0.1 & 5.73 & 9.18 & \\
\hline
\end{tabular}

Table 9. Effect of $b / h$ ratio

\begin{tabular}{|c|c|c|c|c|c|c|}
\hline \multirow{2}{*}{$b$} & \multicolumn{2}{|c|}{ ADI } & \multicolumn{2}{c|}{ Non-ADI } & \multicolumn{2}{c|}{} \\
\cline { 2 - 7 } & Cost & Fill rate & Cost & Fill rate & $\begin{array}{c}\text { Cost savings due to } \\
\text { ADI }\end{array}$ & Fill rate change due to ADI \\
\hline 0.1 & 0.93 & $37.07 \%$ & 0.83 & $49.47 \%$ & $10.84 \%$ & $33.44 \%$ \\
\hline 0.5 & 3.15 & $61.38 \%$ & 2.39 & $60.42 \%$ & $24.25 \%$ & $-1.57 \%$ \\
\hline 1 & 4.97 & $79.99 \%$ & 3.79 & $74.04 \%$ & $23.70 \%$ & $-7.44 \%$ \\
\hline 1.1 & 5.25 & $82.95 \%$ & 4.04 & $74.04 \%$ & $23.07 \%$ & $-10.75 \%$ \\
\hline 1.5 & 6.22 & $87.54 \%$ & 4.84 & $84.97 \%$ & $22.28 \%$ & $-1.73 \%$ \\
\hline 2 & 7.18 & $90.80 \%$ & 5.59 & $89.23 \%$ & $22.17 \%$ & $1.61 \%$ \\
\hline 2.5 & 7.99 & $90.80 \%$ & 6.14 & $92.26 \%$ & $23.14 \%$ & $-0.46 \%$ \\
\hline 3 & 8.69 & $92.69 \%$ & 6.65 & $92.26 \%$ & $23.52 \%$ & $-0.70 \%$ \\
\hline 4 & 9.79 & $95.29 \%$ & 7.43 & $94.62 \%$ & $24.10 \%$ & $0.09 \%$ \\
\hline 5 & 10.61 & $96.41 \%$ & 8.08 & $96.49 \%$ & $23.92 \%$ & $-0.35 \%$ \\
\hline 10 & 13.25 & $98.53 \%$ & 10.21 & $98.18 \%$ & $22.94 \%$ & \\
\hline
\end{tabular}

